\title{
Collective behavior of coupled map lattices with different scales of local coupling
}

\author{
SHI WenJun \\ Department of Physics and Electrical Engineering, Ningde Normal University, Ningde 352100, China
}

Received April 9, 2011; accepted June 7, 2011

In this paper, I present a numerical study on the collective behavior of one-dimensional coupled map lattices with the nearest coupling to different scales for the whole system. Using the maximum Lyapunov exponent as a tool for subsystem and return mapping, I observed several basic patterns of collective behavior and investigated the contrasts between the different scales. To study the mechanism, the system under entirely random perturbations was investigated using the Monte Carlo method and the contrast with the deterministic approach is given. The results show that the response to a random input is complicated and involves the correlation of different signals and taking into consideration the dynamic properties of the system itself.

subsystem, maximum Lyapunov exponent, different scale, return map, random perturbation

Citation: Shi W J. Collective behavior of coupled map lattices with different scales of local coupling. Chinese Sci Bull, 2011, 56: 3723-3730, doi: $10.1007 / \mathrm{s} 11434-011-4667-9$

Space-time chaos has been studied for many years and is still a major topic of research. Using coupled map lattices (CMLs) as a basic model for studying space-time chaos has received increased attention for some time [1]. Previous research on this model has mainly been applied to global dynamics, while little consideration of the dynamics has been given to different scales of the system as the lattices were assumed to be coarse grained. However, if the system has some collective behavior, the dynamics for different scales of the system should be considered. On the other hand, the relationship between few and many bodied systems continues to attract great interest. A similar pertinent question is whether I can also treat the dynamics of the CML with a deterministic approach and add random perturbations? An interesting paper [2] gives a positive answer to this for a special case, although random noise is artificially added. From the research presented in this paper, it is clear that the question is a difficult one. Furthermore, recent works related to nontrivial collective behavior has attracted wide attention. In particular, using Lyapunov modes gives a

email: swjyeah@mail.ustc.edu.cn very new and profound comprehension of collective spatiotemporal chaotic behaviors [3].

In this paper, I aim to carry out a numerical study of collective behaviors for various local scales of the whole system (the scale here is the length of a subsystem). Common and extensively studied models (CML) are employed.

\section{A brief introduction of study model}

The model CML consists of many lattices coupled with each other and each lattice has its own internal dynamics. The internal dynamics are chaotic or periodic according to different internal dynamic parameters. In this paper, only the one-dimensional and nearest coupling are given consideration. The mathematical formula for one-dimensional coupled map lattices is

$$
x_{n+1}(i)=(1-\varepsilon) f\left(x_{n}(i)\right)+(\varepsilon / 2)\left[f\left(x_{n}(i-1)\right)+f\left(x_{n}(i+1)\right)\right],
$$

where $n=1,2, \ldots N$ are discrete time steps, $i=1,2, \ldots L$ are 
discrete lattice sites with periodic boundary conditions $x_{n}(L+1)$ $=x_{n}(1)$ and $x_{n}(0)=x_{n}(L), x_{n}(i)$ represents a continuous state, $\varepsilon$ is the diffusive coupling strength with the nearest neighbor sites, and $f(x)$ governs local dynamics, for $f(x)=1-a x^{2}$ with $a \in[0,2]$ and $x \in[-1,1][4]$.

Based on the research of Kaneko [1], I chose six types of dynamic modes for the global system to study: frozen random pattern, pattern selection, defect chaotic diffusion, defect turbulence, pattern competition intermittency, and fully developed turbulence characterized generally by the spaceamplitude plot and space-time diagram, and called modes 1 , $2,3,4,5$ and 6, respectively, for brevity with $L=100$. Without declaring time steps for evolution, the transient process for evolution cannot be considered. In this study, I selected fixed parameters $(\varepsilon, a)$ for modes $1,2,3,4,5$ and 6 corresponding to $(0.15,1.52),(0.10,1.66),(0.10,1.79),(0.10$, $1.885),(0.30,1.775)$ and $(0.30,2)$.

\section{Numerical analysis}

\subsection{Spatial correlation and statistical equivalence of each lattice}

The spatial correlation of different locations in extended space is employed to investigate dynamic effects of any two lattices in the model studied. For a fixed time $n$, the arithmetic average of a lattice for the whole system is $\langle x\rangle_{n}=$ $(1 / L) \sum_{i=1}^{L} x_{n}(i)$ and the corresponding deviation for this average is $\hat{x}_{n}(i)=x_{n}(i)-\langle x\rangle_{n}$, and thus we write the spatial correlation function, which characterizes the correlation between the different individual lattices as [5]

$$
C_{n}(l) \equiv \frac{(1 / L) \sum_{i=1}^{L} \hat{x}_{n}(i) \hat{x}_{n}(i+l)}{(1 / L) \sum_{i=1}^{L}\left(\hat{x}_{n}(i)^{2}\right)} .
$$

The spatial correlation generally tended to decrease rapidly when the length between two given lattices increased but the time steps for evolution cannot lead to an increased correlation. Numerical studies show that mode 3 and mode 5 have abnormal values. The evolution can strengthen the correlation although the decay of correlation according to increasing length remains. This is a type of long-rang correlation that originated from local coupling. The numerical work is depicted in Figure 1.

To testify the equivalence of every single lattice for its statistical property, I studied the difference between the state value of every single lattice and the arithmetic average of the state values for the whole system under the arithmetic average of a few time steps and a few systems selected at random for initial state values. The transient process for evolution is deleted, and the time and ensemble used
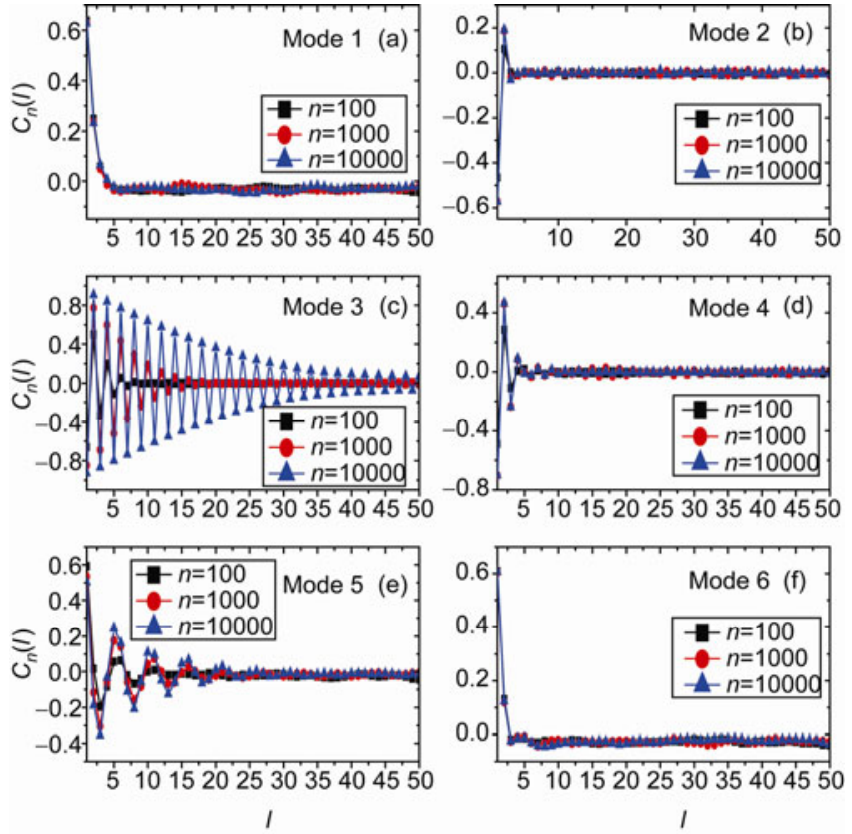

Figure 1 (Color online) Spatial correlation function versus different lengths between two lattices for given steps of time $n=100, n=1000$ and $n=10000$ by averaging 500 random initial conditions. (a)-(f) correspond to modes 1 , 2, 3, 4, 5 and 6 . Mode 3 and mode 5 show abnormal values. The evolution can strengthen the correlation although the decay of correlation according to increasing length remains.

correspond to an order larger than 100. Figure 2 shows, as expected, that every lattice in the model is equivalent, because the system CML is a ring in the periodic condition. Therefore, I studied the dynamic property of a given scale only for lattices of a fixed subsystem but with different initial conditions. For example scale 5 corresponds to a subsystem composed of a fixed lattice $i=1$ to fixed lattice $i=5$ but with different initial state values.

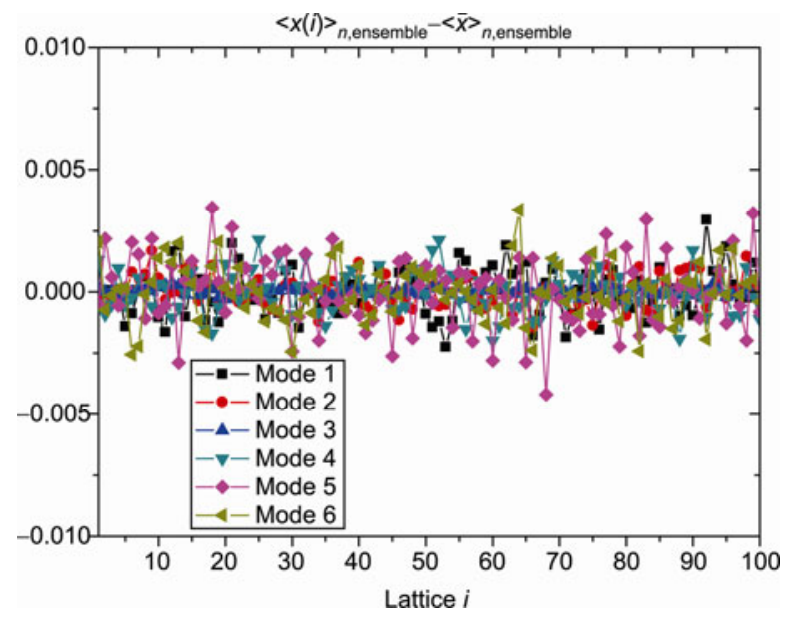

Figure 2 (Color online) Difference between the state value of a single lattice and the arithmetic average of state values for the whole system under the arithmetic average of 100 time steps and 500 systems selected at random with initial state values. The transient time steps are 5000 for the six dynamic modes studied. 


\subsection{Maximum Lyapunov exponent for subsystems}

The stability of the subsystem is now investigated and one of the most important parameters characterizing the dynamic instability of chaotic systems is the spectrum of Lyapunov exponents $[4,6,7]$. Furthermore, there are other characteristic methods for chaotic systems with important applications [8,9]. In particular, the positive maximum Lyapunov exponent (MLE) is an important feature for describing the sensitive dependence on initial conditions for chaotic motion, and thus MLE must be computed frequently. There are two computational methods conventionally accepted as being equivalent. The first method computes the divergence rate of two adjacent trajectories of the system, where the distance between the two trajectories is frequently reformed to a numerical value which is much smaller than the attractor scale and much larger than the computation error. The second is to work in the tangent space of the dynamic system. All Lyapunov exponents are calculated by evaluating the Jacobian of the dynamic function of a system along a trajectory [4].

We see the commonality of the above two methods in that they are both required to compute the average of the divergence rate from a long and true trajectory. For highly dimensional dynamic systems, such as CML, using the first method to compute the MLE is much more direct and has high efficiency, so this method is employed. The basic method to compute the MLE for the whole system is as follows: if I apply a very small perturbation $\left\{\mathrm{d} x_{0}(i)\right\}$ to the state of system $\left\{x_{m}(i)\right\}$, there will be a new state $\overline{x_{m}(i)}=$ $x_{m}(i)+\mathrm{d} x_{0}(i), i=1,2, \ldots L$. Now, iterating CML, I obtain $\left\{x_{m+n}(i)\right\}, \overline{\left\{x_{m+n}(i)\right\}}$ and $\left\{\mathrm{d} x_{n}(i)\right\}$ in the discrete time $m+n$. Therefore, the mathematical formulation of the MLE is

$$
\lambda_{\mathrm{MLE}}=\lim _{N \rightarrow \infty} \frac{1}{N} \sum_{n=1}^{N} \log \frac{\left\|\mathrm{d} x_{n}\right\|}{\left\|\mathrm{d} x_{n-1}\right\|},\left\|\mathrm{d} x_{n}\right\|=\sqrt{\sum_{i=1}^{L}\left[\mathrm{~d} x_{n}(i)\right]^{2}} .
$$

To avoid excessively large perturbations, it should be reiterated for every time step of the evolution.

In this study, the interest is not to focus on the MLE of the whole system but on the MLE of different scales (length of the lattice system). The whole system is divided into two parts: the subsystem and surrounding regions. It is similar to a deterministic system subjected to noise perturbations [10]. We call this the quantity characterizing the subsystems with scale-limited Lyapunov exponents. The corresponding MLE is called the maximum Lyapunov exponent for subsystems (SMLE). The analytical study of SMLE for CML with the nearest coupling can be done for a case that is easily treated, such as a fully chaotic logistic map [11]. General studies of SMLE for different scales of CML are not found in the current literature.

From the numerical simulation depicted in Figure 3, there is a clear tendency of pattern transition from a discontinuing variation to a smooth variation in the SMLE versus
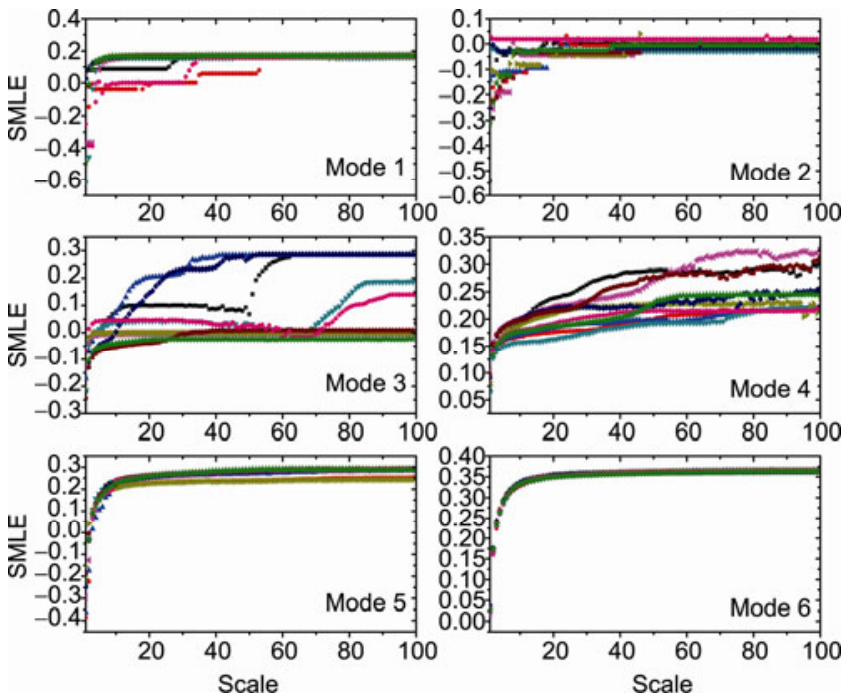

Figure 3 (Color online) Variation in SMLE versus different scales with different types of dynamic modes, 10 random chosen initial state values were taken for each mode. There is a clear tendency for pattern transition from a discontinuing to smooth variations in SMLE as the scale is increased.

increasing scale for all modes with the most random selected initial values. The case of the discontinuing platform of the SMLE is clearly emergent in modes 1 and 2 . Furthermore, the platform is not emergent randomly in different positions. It was found that there were fixed platforms for different random initial state values although I was not able to establish what the exact conditions should be for the emergence of these platforms. The different final states of the system for asymptotic evolution corresponding to different initial states are observed directly for modes 2, 3, 4 and 5 with different values for SMLE. This is a typical feature of high dimensional systems [1].

\subsection{Return map for collective behavior of subsystem versus SMLE}

To study the collective behavior directly, the return map is employed [12,13], and the relationship between the SMLE and return map is compared. The arithmetic average of the state value of every lattice in the subsystem is expressed as

$$
h_{n}=(1 / \text { scale }) \sum_{i=1}^{\text {scale }} x_{n}(i)
$$

and thus the return map is taken as $h_{n+1}$ versus $h_{n}$. I obtain the information of collective behavior from the return map which actually comes from the similar idea of the Poincare map [4]. Unexpectedly, during the process of transition between different platforms of the SMLE, the return map could not be changed sharply but had some variations in the topology structure for most cases from the extensive numerical simulations.

For platforms of the SMLE emerging mainly in modes 1 and 2, typical comparisons between the return map and the SMLE are depicted in Figure 4. As the scale increases, the 


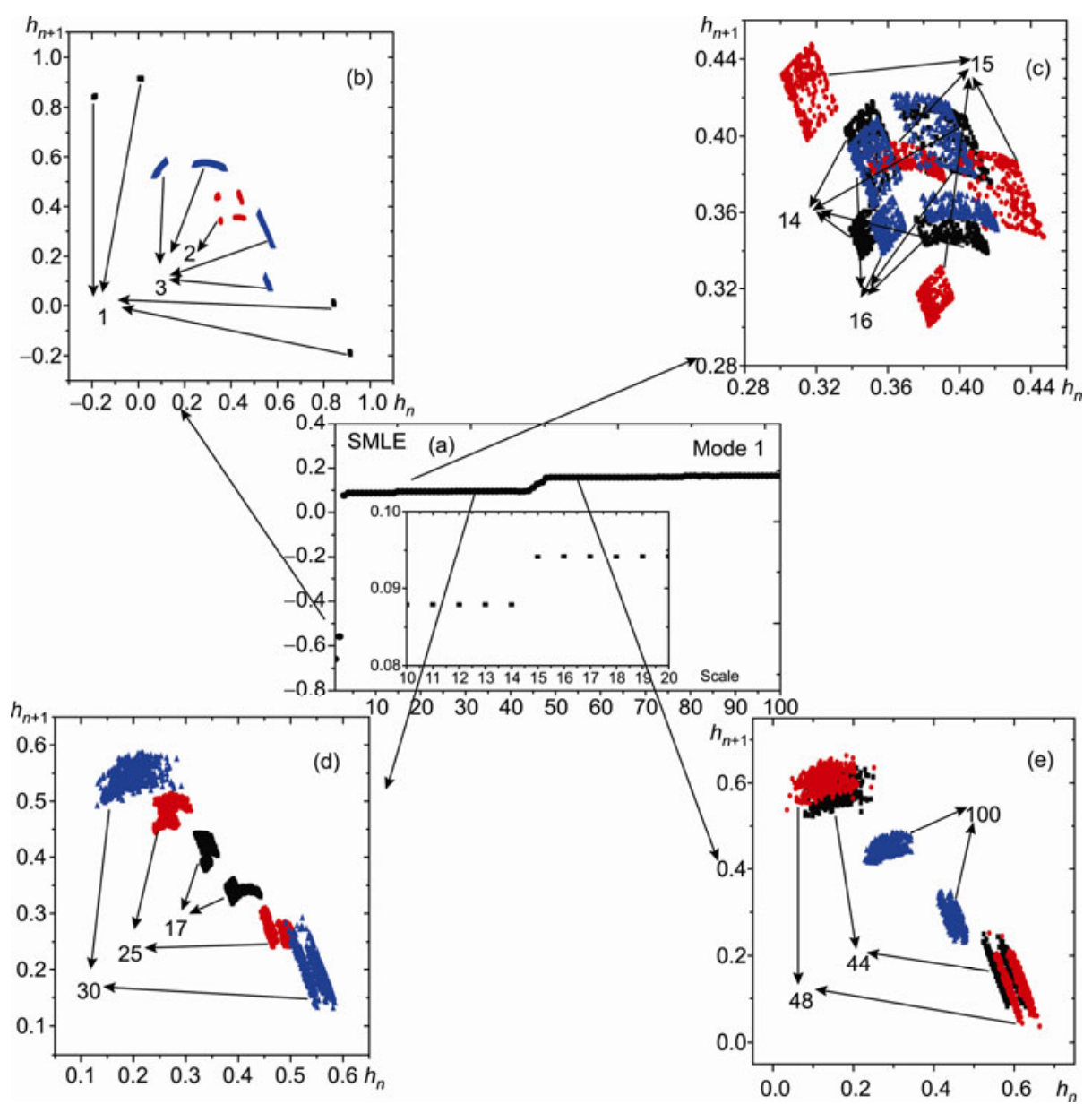

Figure 4 (Color online) A typical return map of the mode 1 with different scales to illustrate the pattern transitions between the discontinuous platforms of the SMLE. (a) gives the variation of SMLE versus different scales, and the inset illustrates the transition of platform of SMLE in detail corresponding to scale $=14$ and 15. (b) shows the change of return map for initial scale $=1,2$ and 3, and the SMLE of scale $=1$ and 2 are negative. (c) shows the change of return map for scale $=14,15$ and 16 corresponding to a discontinuous platform of SMLE as well as the similar contrast of return maps in (e) to a discontinuous platform of SMLE with starting scale $=44$, the return map of scale $=48$ and 100 are also plotted for comparison. (d) shows the change of return map for different scales but corresponding to the same platform of SMLE. The arrows in the figure just give the positions of SMLE corresponding to return maps of different scales.

complexity of the collective behavior also increases. We observe some isolated points depicted in the return map corresponding to negative values for the SMLE. Furthermore, the platforms of SMLE are also numerically observed to be near zero, computed using random initial values, and corresponding return maps show closed curves, i.e. quasiperiodicity (this case is not shown in Figure 4). Return maps to different platforms of a positive SMLE show abnormal changes with some different topological structures although some cases are large and some minor. These situations are illustrated in Figure 4(c) and (e). Meanwhile, for the same platform of the SMLE, the structure of the return map is changed gradually as the scale is increased. Figure 4(d) gives a clear illustration of this transition pattern. The SMLE of mode 2 for most cases is negative which corresponds to the periodicity of $h_{n}$. Some limited points emerge in the return map as expected, similar to the case of Figure 4(b) of mode 1 for scale 1 corresponding to large negative values of SMLE.

The dynamic properties of different scales for mode 3 have special features, and the main is the different structure of the return map with an odd- or even-numbered scale. The structures of the return map with an odd-numbered scale are composed of two separate regions. However, with an even-numbered scale there is only a single region. This is a general rule and there are no exceptions for the numerical simulations illustrated in Figures 5 and 6 for the two typical cases selected. With an increase in SMLE, the different structures for these two patterns gradually merge into the same pattern. After the SMLE introduces the platform shown in Figure 5(a), the pattern cannot be changed further whatever the scale of the subsystem is. Extensive numerical simulations show that the merging process always exists with the exception of the scale corresponding to the last platform of SMLE (in fact the SMLE here has only one or two platforms). 

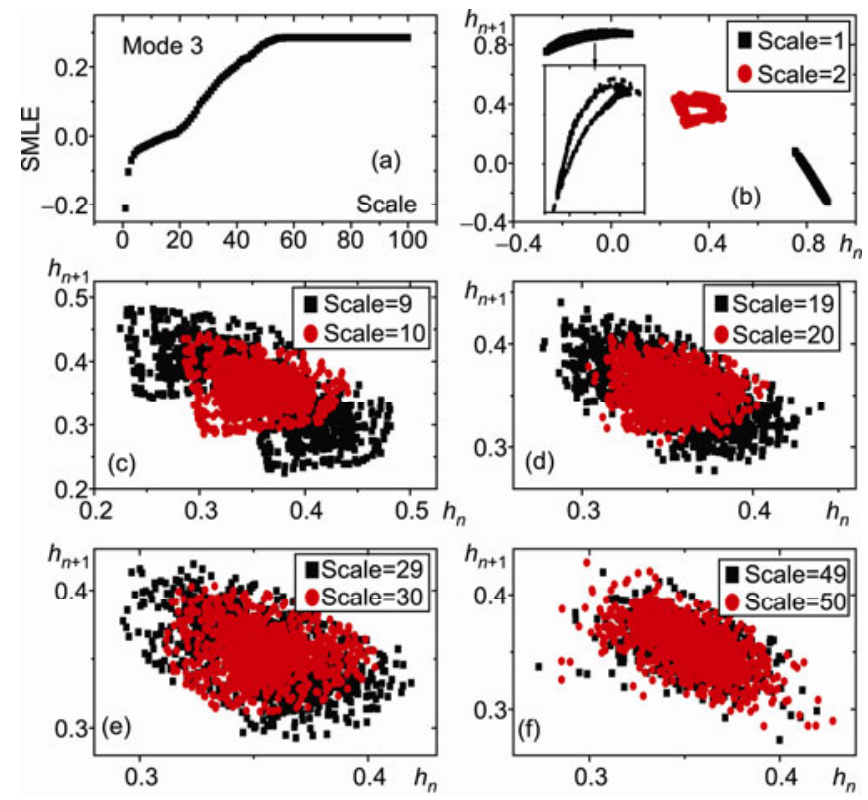

Figure 5 (Color online) Comparisons between the SMLE and return map with different scales for mode 3 corresponding to the typical case of SMLE changed from negative to positive value with the enlargement of scales. (a) shows the variation of SMLE versus the enlargement of scales, (b)-(f) give the illustration of the change of return map with the enlargement of scales. Note that there is a different structure for the return map with an odd- or even-numbered scale, and the structures of the return map with an oddnumbered scale are composed of two separate regions.
A more difficult case showing dynamic variations in the return map of mode 3 is seen in Figure 6 although the initial expression is similar to Figure 5. It is seen that a different pattern of the return map corresponding to an odd- or evennumbered scale always exists but the tendency weakens as the scale increases. The imperfect torus in the return map, with some single points inside it, is broken when the scale is gradually increased, but it does not have the tendency to change to the chaos illustrated in Figure 5. The broken process is also an organized process. Finally I was able to establish a return map for the largest scale, the scale $=100$, which shows the perfect torus, i.e. quasi-periodicity. The case of a scale $=99$ is very similar to perturbations of a Hamiltonian system meeting the condition of integrability, i.e. the crossing of the elliptic points and hyperbolic points is the requirement based on the KAM theory [4].

Dynamic properties for the typical case of mode 4 are illustrated in Figure 7. These share the important characteristics of mode 3 reported above, especially for the different pattern of the return map corresponding to whether the scale is odd or even, although this is not clear for mode 4 with some of the random initial values selected. The top of Figure 7 shows variations in the regions occupied in the return map. There is a clear tendency to decrease the size of the return map although the size changes very slowly. This situation is similar to Figure 6. Careful examination of the
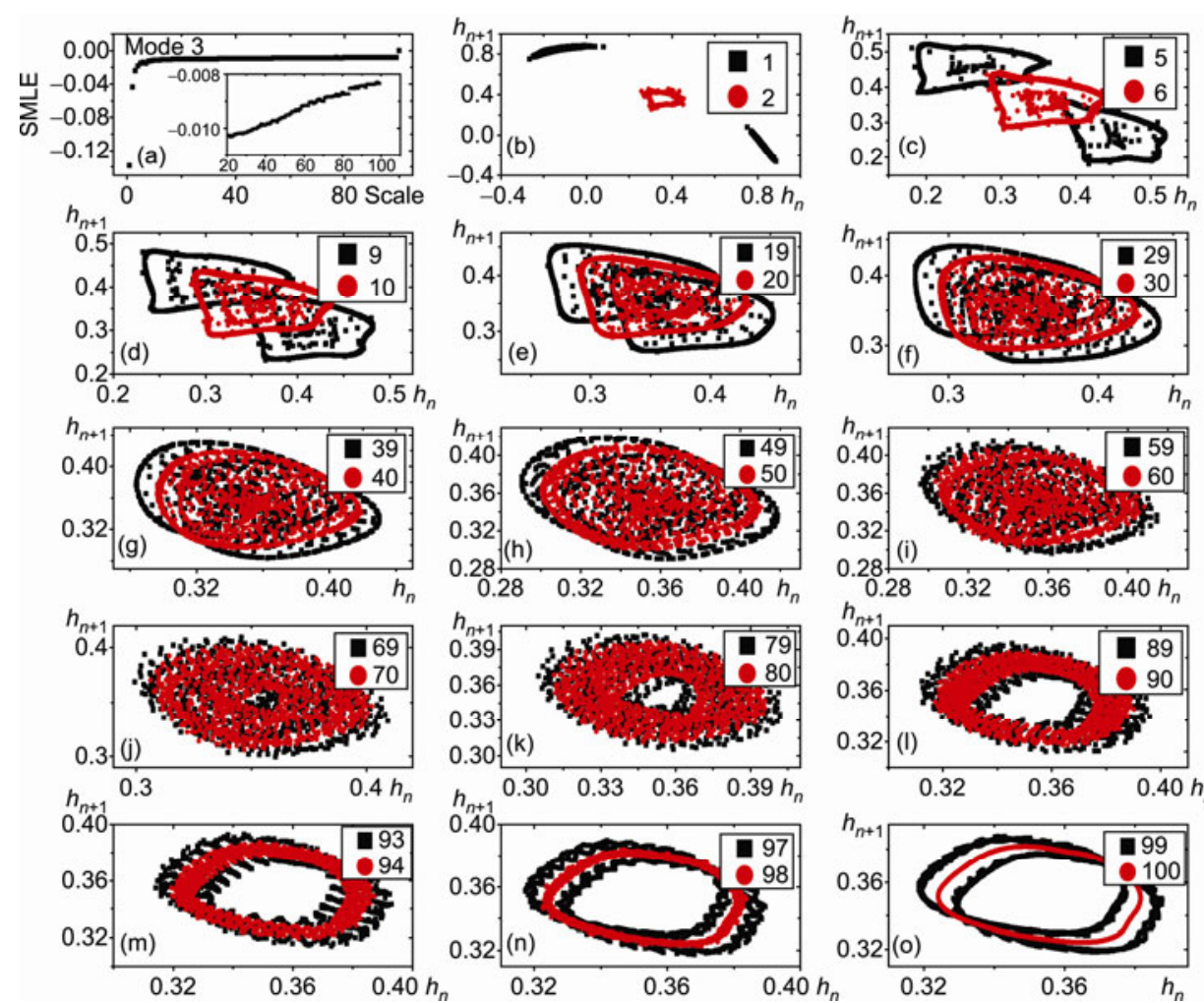

Figure 6 (Color online) Comparisons between the SMLE and return map with different scales for mode 3 corresponding to the typical case of negative SMLE. (a) shows the variation of SMLE versus the enlargement of scales, (b)-(o) give the illustration of the change of return map with the enlargement of scales. Note that the similar situation like Figure 5 of different structures for the return map with an odd- or even-numbered scale, and the typical patterns of return map are weakened with the merging process going on. To note specifically, the broken process of the geometric structure of the return map is also an organized process for the new geometric structure as well. 

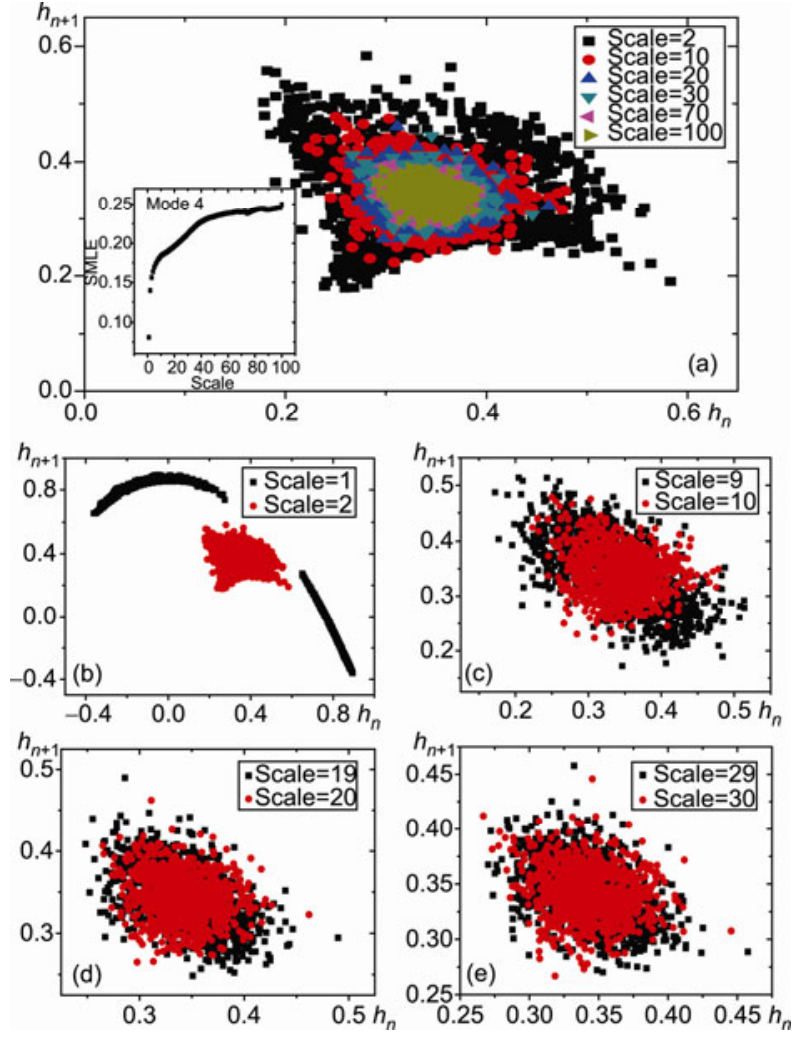

Figure 7 (Color online) Comparisons between the SMLE and return map with different scales for mode 4 . The different structure of the return map with the scale for an odd or even number is held until after the merging process. (a) shows the tendency for return maps to different scales, and some typical scales are selected for this illustration. To show the merging process for the return map with an odd- or even-numbered scale, (b)-(e) show the process.

figures reveals the common feature of increasing the SMLE corresponds to decreasing the size of the return map. The inset in Figure 6(a) clearly illustrates this. Based on the investigation above, I conclude that dynamic modes 3 and 4 share the same class of collective behaviors for their subsystems.

Modes 5 and 6 share the common feature illustrated clearly in Figure 8, where all the regions of the return map for different scales of subsystems are located in the region surrounded by the region of the return map for the scale $=1$ with greater symmetry for mode 6 . Conversely, the size of the region occupied in the return map decreases as the scale of the subsystem increases and the corresponding SMLE increases. Figure 8(a) and (b) clearly illustrates this.

Careful study of the SMLE and return map, reveal the basic patterns for the collective behaviors of the subsystems. This raises several questions. First, I note that the traditional methods for studying the relationship between different lattices, such as the spatial correlation function, cannot directly characterize the collective behaviors. While the expressions for the spatial correlation of mode 3 and mode 4 are quite different, they share the same class of collective behaviors as in the analysis above. Therefore, combining
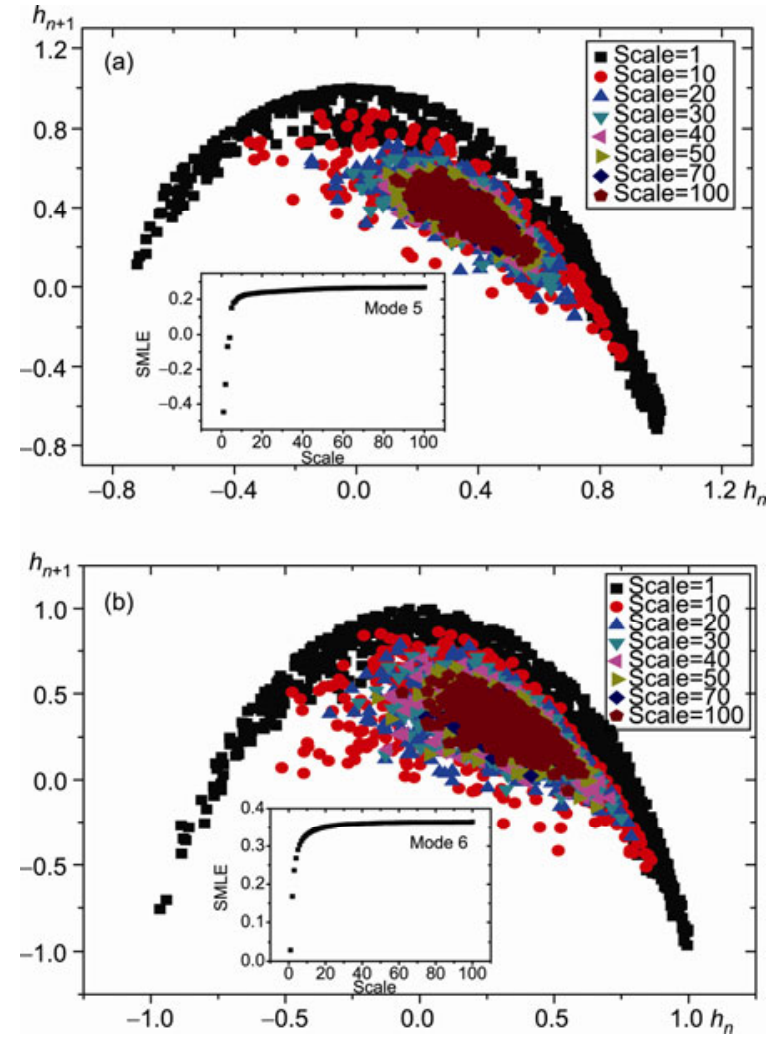

Figure 8 (Color online) Comparisons between the SMLE and return map with different scales for typical cases of mode 5 (a) and mode 6 (b). All regions of the return map for different scales of subsystems are located in the region of the return map for scale $=1$, and there is a greater degree of symmetry for mode 6 compared with that of mode 5 . The size of the region occupied in the return map decreases as the scale of the subsystem increases and as the corresponding SMLE increases.

the SMLE and return map, the emergence of global attractors is observed. Furthermore, these methods can be extended to more real physical systems with space extended beyond the CML. This enables the investigation of the collective behaviors for different scales of subsystems, such as many-particle Hamiltonian systems.

\subsection{Connection with random dynamics using maximum Lyapunov exponent}

We now consider the likely connection with random dynamics. The dynamic behavior of the subsystem can be considered from two perspectives: the deterministic and the random. The subsystem, subject to the effect of the evolution of the whole system, is equivalent to an independent system with perturbations from the boundary lattices. For the model studied, $x(L)-x($ scale $)$ and $x($ scale +1$)-x(1)$ correspond to perturbations of $x(1)$ and $x$ (scale), and $L$ is the length of the whole system. We also investigated the equivalence of deterministic dynamics of the whole system and a few of the dynamics of the body subject to random forces. Recent study [14] is relevant to this work. The research in this paper is different from that paper because random 
samples are obtained from the lattice system itself rather than using artificially added random samples. To justify the assumption of equivalence, the Monte Carlo method is employed to study the stability of the lattice system with random perturbations generated from the whole original system using a numeric statistical distribution [15]. The samples used are statistically independent and the typical discrepancies of the SMLE between them are depicted in Figure 9.

To differentiate the independent system with random perturbations from the subsystem under the evolution of the whole system, I use $\lambda_{\max }$ to express the MLE common to these two different types of system. The transient process for evolution is deleted and initial state values for the numerical computation of the two types of system are the same. From the numerical study, the results cannot be interpreted by spatial correlation, as the intuitive assumption that the less the correlation between lattices the more coincidence in $\lambda_{\max }$ between the random and the deterministic systems, is not supported.

It is possible that there is an important role for the weak correlation of perturbations introduced from the two lattices on the boundary to the dynamic stability, i.e. $\lambda_{\max }$. A strong oscillation of $\lambda_{\max }$ for the random system in modes 3 and 4 versus an odd- or even-numbered scale was found. Furthermore this oscillation coincides with a different pattern for the return map with the scale of the subsystem for even or odd numbers. Although in Figure 9 the random system of mode 2 expresses almost the same values of $\lambda_{\max }$ as for the deterministic system, there have been some large deviations in some of the lengths of the system. For a relatively large length of the system, there has been a trend to the same
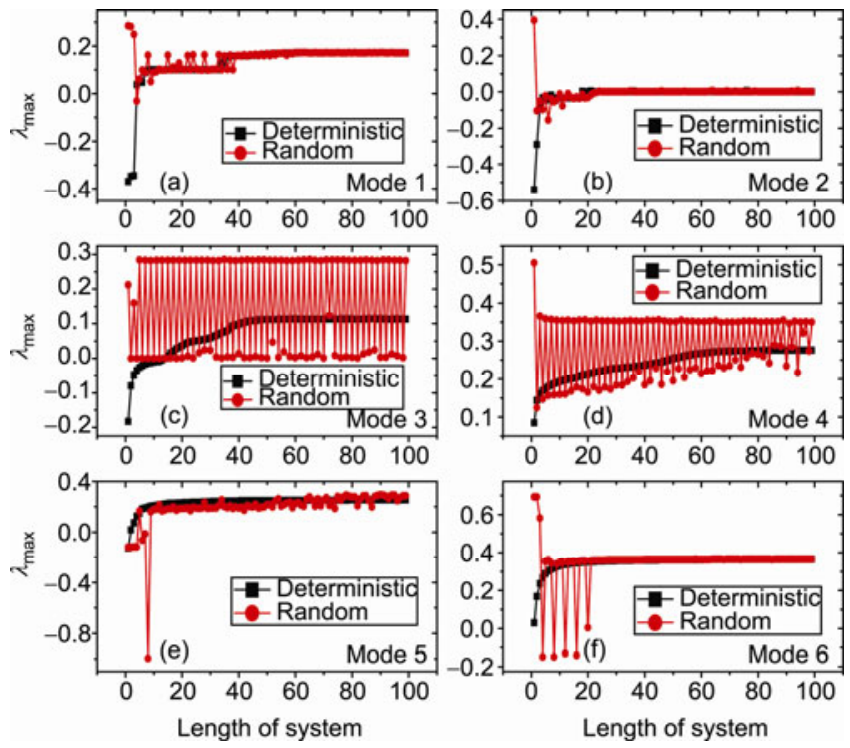

Figure 9 (Color online) Comparison of typical cases of six modes with the largest Lyapunov exponents between a system with random perturbations generated by the distribution of probabilities obtained from the boundary lattices in the sense of whole system's evolution (round box) and the corresponding deterministic system (SMLE) (square box). value of $\lambda_{\max }$ between the random and deterministic systems, which corresponds to the decreasing spatial correlation. However, it is clear that the mechanism of the numerical results is still an ongoing problem.

\section{Discussion and conclusions}

This paper considers the stability of a subsystem subject to the evolution of the whole system. The numerical analysis of collective behaviors of subsystems is the focus, with the spatial correlation function between two lattices taken as a complementary tool to study complexity. The pattern for collective behavior has a relationship that corresponds with the stability of the subsystem. This paper also studies the comparison of the random system with samples obtained from the boundary lattices and the subsystem subject to the evolution of the whole system. The results show that an entirely random system cannot be equivalent to a deterministic one from correlation between lattices. The mechanism for the collective behaviors is still an ongoing problem, and the propagation of perturbations being accounted for in the expression of SMLE [16] remains for future study. Furthermore, the study under the Hamiltonian frame for treating perturbations needs serious consideration [17]. The relationship between random perturbations and collective behavior is also an interesting topic with promising application [18].

Another related case of interest is to consider different types of local dynamics and different types of ranges for coupling [19-28] in investigating the variations in patterns of collective behaviors as well as the SMLE. Considering the non-extensivity of the long-range coupling case, nonextensive statistical mechanics may be applied for a deeper understanding of numerical results [29]. A nontrivial collective behavior-synchronization of whole lattices was found when the coupling value surpassed some critical value for system's other fixed parameters [30], thus posing an interesting question as to whether there are non-trivial relationships between the synchronization and topological structure of the return map as well as for the SMLE (for synchronization, the return map for each scale of subsystem is the same).

In summary, this paper first takes the SMLE and return map as main tools for studying collective behaviors of the different scales of subsystems, although extensive studies were also taken for the CML with the nearest coupling. Using numerical simulation, I obtained new results not previously reported and found basic patterns for collective behaviors. Therefore, this study has value in searching for the connection of dynamics between few and many bodies [31, 32]. We expect more valuable achievements in the future.

The author thanks Professor Beck Christian of London University for helpful modification of the abstract and introduction. The work was supported by the Fujian Provincial Natural Science Foundation (2007J0205) 
and the Project of Scientific Research of Ningde Normal University (2006Y007).

1 Kaneko K. Theory and Application of Coupled Map Lattices. New York: Wiley Press, 1993

2 Carretero-González R, Ørstavik S, Huke J, et al. Thermodynamic limit from small lattices of coupled maps. Phys Rev Lett, 1999, 83: 3633-3636

3 Takeuchi K A, Ginelli F, Chate H. Lyapunov analysis captures the collective dynamics of large chaotic systems. Phys Rev Lett, 2009, 103: 154103

4 Ott E. Chaos in Dynamic Systems. London: Cambridge University Press, 2001

5 Vasconcelos R B, Viana R L, Lopes S R, et al. Spatial correlations and synchronization in coupled map lattices with long-range interactions. Physica A, 2004, 343: 201-218

6 Wang Z Z, Li Z, Wei Y X, et al. Lyapunov exponents for synchronous 12-lead ECG signals. Chinese Sci Bull, 2002, 47: 1845-1848

7 Wang N, Ruan J. Principal component cluster analysis of ECG time series based on Lyapunov exponent spectrum. Chinese Sci Bull, 2004, 49: 1980-1985

8 Li J, Ning X B. The base-scale entropy analysis of short-term heart rate variability signal. Chinese Sci Bull, 2005, 50: 1269-1273

9 Wang X Y. Relation of chaos activity characteristics of the cardiac system with the evolution of species. Chinese Sci Bull, 2002, 47: 2042-2048

10 Pecora L M, Carroll T L. Driving systems with chaotic signals. Phys Rev A, 1991, 44: 2374-2383

11 Carretero-González R, Ørstavik S, Huke J, et al. Scaling and interleaving of subsystem Lyapunov exponents for spatio-temporal systems. Chaos, 1999, 9: 466-482

12 Shibata T, Chawanya T, Kaneko K. Noiseless collective motion out of noisy chaos. Phys Rev Lett, 1999, 82: 4424-4427

13 Monte S D, Ovidio F, Chaté H, et al. Noise-induced macroscopic bifurcations in globally coupled chaotic units. Phys Rev Lett, 2004, 92: 254101

14 Santos A M, Viana R L, Lopes S R, et al. Collective behavior in coupled chaotic map lattices with random perturbations. Physica A, 2008, 387: $1655-1668$

15 Landau D P, Binder K. A Guide to Monte Carlo Simulations in Statistical Physics. London: Cambridge University Press, 2000

16 Saarloos W V. Front propagation into unstable states. Phys Rep, 2003,
386: 29-222

17 Chen L C, Zhu W Q. First passage failure of dynamical power systems under random perturbations. Sci China Tech Sci, 2010, 53: 2495-2500

18 Ma J, Wu Y, Ying H P, et al. Channel noise-induced phase transition of spiral wave in networks of Hodgkin-Huxley neurons. Chinese Sci Bull, 2011, 56: 151-157

19 Torcini A, Lepri S. Disturbance propagation in chaotic extended systems with long-range coupling. Phys Rev E, 1997, 55: R3805-R3808

20 Pinto S E, Viana R L. Synchronization plateaus in a lattice of coupled sine-circle maps. Phys Rev E, 1999, 61: 5154-5161

21 Batista A M, Viana R L. Lyapunov exponents of a lattice of chaotic maps with a power-law coupling. Phys Lett A, 2001, 286: 134-140

22 Batista A M, Pinto S E, Viana R L, et al. Lyapunov spectrum and synchronization of piecewise linear map lattices with power-law coupling. Phys Rev E, 2002, 65: 056209

23 Anteneodo C, Pinto S E, Batista A M, et al. Analytical results for coupled-map lattices with long-range interactions. Phys Rev E, 2003, 68: 045202

24 Viana R L, Grebogi C, Pinto S E, et al. Validity of numerical trajectories in the synchronization transition of complex systems. Phys Rev E, 2003, 68: 067204

25 Lind P G, Corte-Real J, Gallas J A C. Inducing coherence in networks of bistable maps by varying the interaction range. Phys Rev E, 2004, 69: 026209

26 Anteneodo C, Batista A M, Viana R L. Chaos synchrozation in longrange coupled map lattices. Phys Lett A, 2004, 326: 227-233

27 Pinto S E, Lunardi J T, Saleh A M, et al. Some aspects of the synchronization in coupled maps. Phys Rev E, 2005, 72: 037206

28 Santos A M, Woellner C F, Lopes S R, et al. Lyapunov spectrum of a lattice of chaotic systems with local and non-local couplings. Chaos Solit Fract, 2007, 32: 702-710

29 Robledo A, Moyano L G. q-deformed statistical-mechanical property in the dynamics of trajectories en route to the Feigenbaum attractor. Phys Rev E, 2008, 77: 036213

30 Szmoski R M, Pinto S E, Kan M T, et al. Synchronization and suppression of chaos in non-locally coupled map lattices. J Phys, 2009, 73: 999-1009

31 Liu Z X, Guo L. Connectivity and synchronization of Vicsek model. Sci China Ser F-Inf Sci, 2008, 51: 848-858

32 Pan C, Wang J J, Zhang C. Identification of Lagrangian coherent structures in the turbulent boundary layer. Sci China Ser G-Phys Mech Astron, 2009, 52: 248-257

Open Access This article is distributed under the terms of the Creative Commons Attribution License which permits any use, distribution, and reproduction in any medium, provided the original author(s) and source are credited. 